\title{
La creatividad como indicador de competitividad empresarial en el panorama publicitario brasileño ${ }^{1}$
}

\section{Criatividade como um indicador de competitividade empresarial no cenário da publicidade brasileira}

Creativity as an indicator of competitiveness business in the brazilian advertising landscape

\author{
Rafael Ravina Ripoll ${ }^{2}$ \\ Estela Núñez Barriopedro 3 \\ Rebeca Álvarez Suárez ${ }^{4}$
}

${ }^{1}$ El presente artículo es resultado de investigación del Grupo de Trabajo Economia Criativa, Aprendizaje y solidariedad ativa in la dinamica territorial, Planteamiento Urbano y Regional.

2 Doctor profesor de Organización de Empresa de la Universidad de Cádiz. Es autor y coautor de artículos, trabajos en congresos internacionales y libros vinculados con la economía de la felicidad y la creatividad. Ha sido profesor invitado en la Universidad Autónoma de Baja California. la Business and Law Frankfurt University of Applied Sciences y la Universidad de Verona, donde ha impartido conferencias sobre el happiness management. Universidad de Cadiz, Espanha.

E-mail: rafael.ravina@uca.es

${ }^{3}$ Doctora en Ciencias Económicas y Empresariales por la Universidad de Alcalá y Licenciada en Administración y Dirección de Empresas en dicha universidad. Ha recibido varios premios en docencia y en investigación. Es Profesora acreditada en UAH, UC3M y UNIR entre otras. Tiene numerosas publicaciones y es directora académica del MBA en la Universidad de Alcalá. Universidad de Alcalá de Henares, Espanha.E-mail: Estela.nunezb@uah.es

${ }^{4}$ Graduada en Administración y Dirección de Empresas por la Universidad de Alcalá de Henares con estancia en la Universidad Hanze de Ciencias Aplicadas (Groningen, Países Bajos). Ha participado como ponente en Congresos Internacionales de Marketing Público y en el Lucrativo. Entre sus publicaciones destaca su capítulo de libro "Cartoon Helpline: una llamada a la ilusión". Universidad de Alcalá de Henares, Espanha.E-mail: rebeca.as.95@gmail.com. 
Resumen: El objetivo de este trabajo es analizar y medirla publicidad en Brasil, especialmente en función del nivel de creatividad de las agencias publicitarias. La metodología de la investigación está basada en la realización del Ranking Iberoamericano de Publicidad en la región de Brasil, que pondera los premios con los que son galardonadas las agencias publicitarias por sus anuncios y los festivales en los que participan. En este trabajo se estudian las mejores cincuenta agencias brasileñas en dicho ranking, analizando su posición y tendencia para los años 2014, 2015 y 2016. Estos años cuentan con una muestra total de 113, 114 y 71 agencias publicitarias respectivamente.

Palabras clave: ranking; creatividad; Brasil; anuncios; agencias de publicidad.

Resumo: O objetivo deste trabalho é analisar e medir a publicidade no Brasil, especialmente em função do nível de criatividade das agências de publicidade em sua propducto ou trabalho de publicidade, os anúncios. A metodologia da pesquisa é baseado na realização do Iberoamericano Ranking Publicidade na região do Brasil, que pondera os prêmios que são concedidos agências de publicidade para seus anúncios e festivais em participar. Neste trabalho os melhores cinquenta agências brasileiras no ranking são estudados, analisando a sua posição e tendência para os anos de 2014, 2015 e 2016. Estes anos têm uma amostra total de 113, respectivamente, 114 e 71 agências de publicidade.

Palavras-chave: ranking; criatividade; Brasil; propagandas; agências de publicidade.

Abstract: The aim of this project is to analyse and measure advertising in Brazil, as well as the companies that form it. The research is based on the Latin American Ranking of Advertising, which weighs awards and festivals both agencies and advertisements. During this work, the best fifty Brazilian agencies of this ranking are studied, analysing their position and trend for the years 2014, 2015 and 2016. These years have a total sample of 113, 114 and 71 advertising agencies respectively.

Keywords: ranking; creativity; Brazil; advertisements; advertising agencies. 


\section{INTRODUCCIÓN}

En un mundo tan internacionalizado como el actual, donde las compañías luchan por ser las más competitivas no sólo en un país si no a nivel mundial, los rankings se han vuelto una herramienta muy útil para medir todo tipo de entidades y compañías, ya que permiten medir y ordenar según un mismo criterio diferentes elementos.

Es por esto que en el último quinquenio la publicación de rankings se ha convertido en un método habitual para determinar la importancia de todo tipo de entidades. El aumento de estas listas ha dado lugar a la creación de un sistema de información sobre rankings (Ranking Systems Clearinghouse apadrinado por el Institute for Higher Education Policy's) lo que promueve las buenas prácticas a la hora de su elaboración por parte de las entidades creadoras.

Un ranking es, una clasificación de mayor a menor que sirve para establecer criterios de valoración mediante un juicio determinado, y en el ámbito publicitario, los rankings deben ser realizados de tal manera que permitan exponer la calidad de las entidades, o agencias publicitarias.

El objetivo de las agencias publicitarias es ocupar las primeras posiciones de los rankings publicitarios, especialmente aquellas agencias pertenecientes a grupos internacionales. La implantación de rankings de publicidad comienza a principios de los sesenta en Norteamérica y Reino Unido con el motivo de jerarquizar a las agencias según sus méritos y logros. La tendencia de usar rankings en este ámbito ha ido creciendo, pasando las fronteras del mundo anglo-sajón y convirtiéndose en una herramienta universal.

Los rankings de publicidad tienen una gran repercusión en las agencias, ya que les dan notoriedad ante los medios de comunicación y aumentan la visibilidad de la marca. Además, estos ayudan a simplificar el complicado sector de la publicidad ante la audiencia.

Repiso y Berlanga (2015) señalan que los rankings de anuncios y de agencias son muy útiles en el sector debido a su espíritu competitivo. Por lo que, los rankings publicitarios más valorados son los que premian la creatividad o la eficiencia publicitaria, dándose en su mayoría más importancia a la creatividad. Este último tipo de rankings son los más 
apreciados por los publicistas, ya que esta cualidad se considera la característica más importante y admirada del sector. Pero no son solo los rankings los que benefician, a la imagen de las agencias. Los festivales de premios publicitarios también suponen prestigio y estatus para las compañías.

Se pueden distinguir varios tipos de rankings de publicidad dependiendo del área geográfica que abarquen (nacional, regional o internacional) o según la valoración que usen. La tabla 1 representa los rankings publicitarios que tienen elaboración anual.

Tabla 1- Rankings de publicidad

\begin{tabular}{|l|c|c|}
\hline \multicolumn{1}{|c|}{ NOMBRE } & ÁREA GEOGRÁFICA & VALORACIÓN \\
\hline ESCO & España & Creatividad \\
\hline Infoadex & España & Facturación \\
\hline UNIR & Iberoamérica & Creatividad \\
\hline Crema de Adlatina & Iberoamérica & Creatividad \\
\hline W \& V-Kreativ & Alemania & Creatividad \\
\hline Ad Age Ranking & USA & Facturación \\
\hline Big won report & Internacional & Creatividad \\
\hline The Holmes Report & Internacional & Facturación \\
\hline
\end{tabular}

Fuente: Elaboración propia.

Para la realización de este trabajo se han elegido dos criterios fundamentales en los que se basa el ranking publicitario. La primera, es la consideración del ámbito geográfico de Brasil. Y la segunda, es la creatividad como indicador de valoración del cada posicionamiento en el ranking. Con respecto al área geográfica, es el único, junto con el ranking Crema de Adlatina, que se centra en el área de Iberoamérica. Sin embargo, la diferencia entre ambos se encuentra en tamaño muestral. Mientras que el ranking Crema solo valora el top ten de cada país, el ranking realizado en este trabajo valora el top 50 con una muestra de113, 114 y 71 agencias publicitarias para los años 2014, 2015 y 2016 respectivamente.

Para medir el nivel de creatividad, este ranking se basa en la ponderación de premios con los que son galardonados las agencias 
publicitarias en cada uno de los festivales. La investigación en creatividad ha tenido una amplia atención en psicología y marketing. Mientras que la mayoría de los estudios desde el punto de vista del marketing se orientan a la efectividad, los estudios psicológicos se enfocan más en medir la creatividad. Destacan, el impacto de la creatividad en los anuncios, en la cuota de mercado, y en como los consumidores perciben esa creatividad entre muchos otros.

En este trabajo se investigan las agencias publicitarias brasileñas, al ser este país uno de los líderes en el sector publicitario del área iberoamericana, así como el país que más invierte en publicidad.

\section{MARCO TEÓRICO}

Brasil es uno de los referentes publicitarios de la región de Iberoamérica por ser el país que más invierte en publicidad (STATISTA, 2017), con un presupuesto de 18.760 millones de dólares durante el año 2014, y alcanzando una cantidad estimada para el año 2019 de 29.000 millones de dólares. Se estima que tendrá un 57\% del total del gasto total publicitario en Iberoamérica para el año 2017. Se prevé que durante los próximos años no solo mantenga el liderazgo del aumento del gasto publicitario en la región, si no que se posicione también en los primeros puestos a nivel global, manteniéndose como uno de los países líderes del sector. Dentro del país, São Paulo es el centro neurálgico de la actividad comercial, así como del sector publicitario (FOREIT; DE CASTRO; FRANCO, 1989).

Siguiendo la tendencia general iberoamericana, el medio en el que más dinero se invierte es la televisión, con un 55\% del gasto total de 2015, seguido de los periódicos con un $13 \%$ y por Internet con un $7 \%$. Brasil ostenta el porcentaje más alto de visitantes diarios a las redes y la mayor cantidad de minutos por visita de la región iberoamericana. En total un 58\% de la población brasileña usa internet a diario, ya sea mediante un ordenador o por la conexión a internet de los teléfonos móviles. Esto desemboca en una previsión de la subida en el gasto en este medio, que se espera siga la tendencia global según anuncia la Asociación Brasileña de Agencias Publicitarias (ASSOCIAÇÃO BRASILEIRA DE AGÊNCIAS DE PUBLICIDADE [ABAP], 2016). 
Si se presta atención al tipo de anunciantes que predominan en Brasil, se pueden diferenciar dos grupos de entidades: públicas y privadas. Se puede observar una clara dominancia en el gasto por parte de las compañías privadas frente a las públicas. En el ámbito de entidades públicas, la lista está principalmente formada por los distintos ministerios del gobierno brasileño, apareciendo además ayuntamientos de ciudades de gran tamaño como Río de Janeiro y São Paulo. Por otro lado, enfocándose en las privadas, se observa que la mayoría son empresas nacionales brasileñas, con excepciones de grandes multinacionales como Unilever, Procter \& Gamble o Fiat. A continuación, la Tabla 2 muestra una lista con las quince compañías que más invierten en publicidad en los dos grupos anteriormente señalados.

Tabla 2- Ranking de anunciantes en Brasil según inversión

\begin{tabular}{|c|c|c|c|c|}
\hline \multirow[b]{2}{*}{ RANKING } & \multicolumn{2}{|l|}{ EMPRESAS PÚBLICAS } & \multicolumn{2}{|c|}{ EMPRESAS PRIVADAS } \\
\hline & Anunciante & $\begin{array}{l}\text { Inversión } \\
\mathrm{R} \$(000)\end{array}$ & Anunciante & $\begin{array}{l}\text { Inversión } \\
\mathrm{R} \$(000)\end{array}$ \\
\hline 1 & Ministerio de Educación & 1.021 .805 & Genomma & 3.951 .605 \\
\hline 2 & Ministerio de Sanidad & 399.115 & ViaVarejo & 3.851 .141 \\
\hline 3 & Gobierno federal & 221.069 & Unilever Brasil & 3.109 .355 \\
\hline 4 & Gobierno estatal de Sao Paulo & 197.064 & Hypermarcas & 2.236 .988 \\
\hline 5 & Ministerio de Defensa & 153.375 & Caixa (GFC) & 1.915 .750 \\
\hline 6 & Ministerio de la Marina & 118.750 & Ambev & 1.463 .305 \\
\hline 7 & $\begin{array}{c}\text { Ayuntamiento municipal de } \\
\text { Sao Paulo }\end{array}$ & 101.479 & $\begin{array}{l}\text { Petrobras } \\
\text { (GFC) }\end{array}$ & 1.331 .478 \\
\hline 8 & $\begin{array}{c}\text { Ayuntamiento municipal de } \\
\text { Río de Janeiro }\end{array}$ & 95.540 & Telefónica & 1.269 .577 \\
\hline 9 & Gobierno estatal de Bahía & 88.457 & $\begin{array}{c}\text { Banco de } \\
\text { Brasil (GFC) }\end{array}$ & 1.037 .666 \\
\hline 10 & Ministerio de Cultura & 74.612 & Fiat & 994.926 \\
\hline 11 & Ministerio Público Federal & 64.408 & $\begin{array}{c}\text { Procter \& } \\
\text { Gamble }\end{array}$ & 975.891 \\
\hline 12 & $\begin{array}{c}\text { Ayuntamiento municipal de } \\
\text { Salvador }\end{array}$ & 52.844 & Itau & 964.787 \\
\hline 13 & $\begin{array}{l}\text { Ministerio de desarrollo So- } \\
\text { cial y lucha contra el hambre }\end{array}$ & 52.121 & $\begin{array}{l}\text { DivcomPhar- } \\
\text { ma Nordeste } \\
\end{array}$ & 938.938 \\
\hline 14 & $\begin{array}{c}\text { Gobierno estatal de Minas } \\
\text { Gerais }\end{array}$ & 46.440 & Bradesco & 886.522 \\
\hline 15 & $\begin{array}{l}\text { Secretaría especial de } \\
\text { políticas para las mujeres }\end{array}$ & 45.890 & Seara & 860.063 \\
\hline
\end{tabular}

Fuente: Elaboración propia. 
Por otro lado, la ABAP realiza un seguimiento anual del gasto en publicidad del país por sectores. Tal y como se puede ver en el Gráfico 3, el primer puesto lo ocupan las empresas de venta al por menor, seguido de empresas de servicio al consumidor y aquellas que ofrecen productos de higiene y belleza. Los últimos puestos los ocupan sectores como el de sorteos de loterías y casas de juego, bienes y servicios industriales y servicios de decoración.

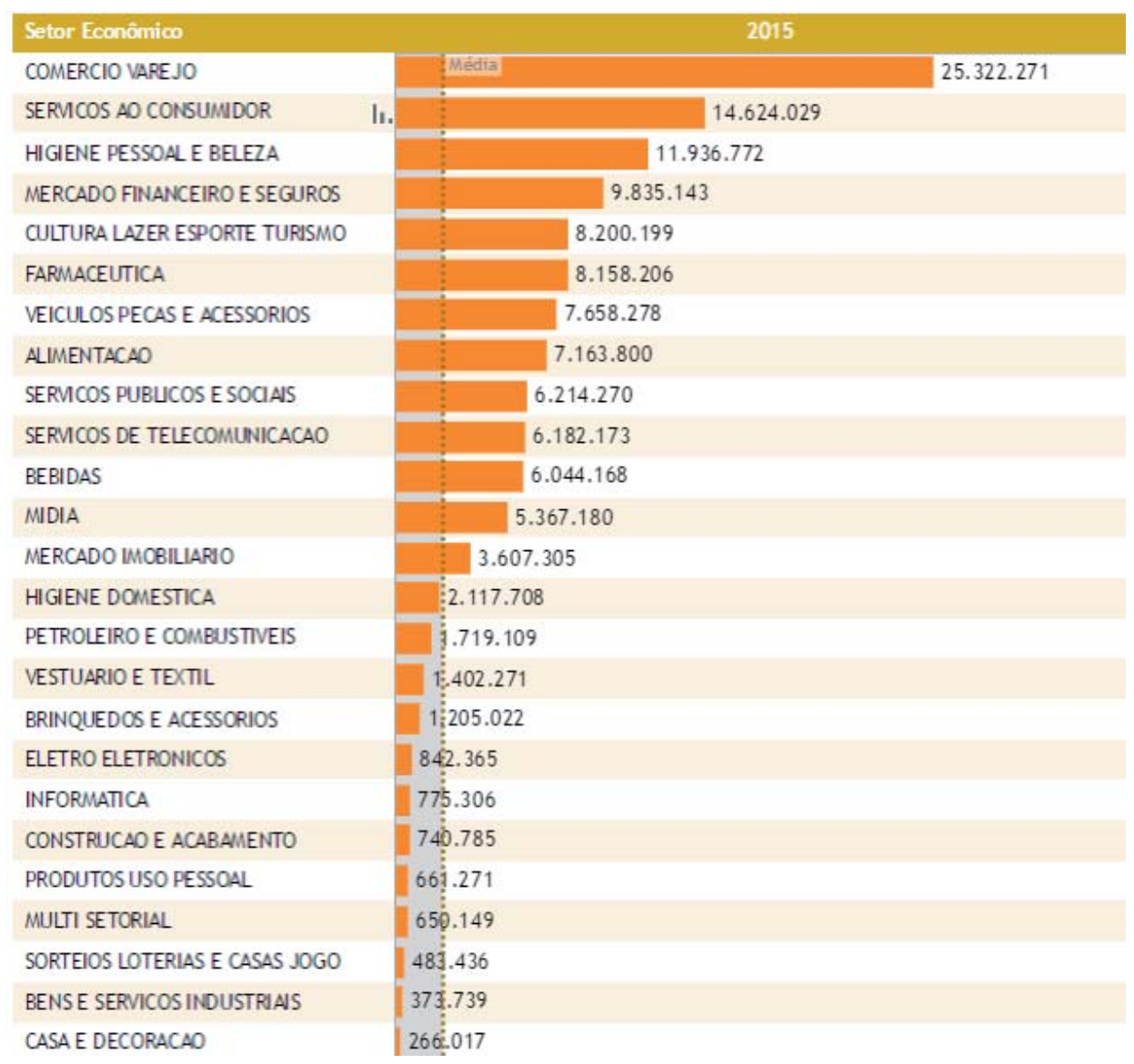

Gráfico 1 - Inversión publicitaria por sector económico (en reales brasileños) Fuente: ABAP (2016). 
Paralelamente se encuentra el tema de la inversión que realizan las empresas de publicidad en Brasil. Tal y como se muestra en la Tabla 4, el top 50 lo forman una clara mayoría de agencias internacionales, como Young \& Rubicam y Ogilvy \& Mather, frente a una minoría de agencias nacionales brasileñas, como por ejemplo Escala Comunicaçao.

Tabla 3 - Ranking de agencias publicitarias en Brasil según inversión

\begin{tabular}{|c|l|c|}
\hline RANKING & \multicolumn{1}{|c|}{ AGENCIA } & INVERSIÓN (R\$) \\
\hline $\mathbf{1}$ & Y\&R & $7.022 .245,44$ \\
\hline $\mathbf{2}$ & OGILVY \& MATHER BRASIL & $3.820 .651,88$ \\
\hline $\mathbf{3}$ & AFRICA & $3.507 .865,15$ \\
\hline $\mathbf{4}$ & WMCCANN & $3.502 .086,21$ \\
\hline $\mathbf{5}$ & ALMAP BBDO & $3.444 .892,70$ \\
\hline $\mathbf{6}$ & LEO BURNETT TAILOR MADE & $3.231 .056,21$ \\
\hline $\mathbf{7}$ & HAVAS WORLDWIDE & $2.825 .378,94$ \\
\hline $\mathbf{8}$ & MULLEN LOWE BRASIL & $2.695 .074,37$ \\
\hline $\mathbf{9}$ & PUBLICIS PBC COMUNICAÇAO & $2.591 .768,85$ \\
\hline $\mathbf{1 0}$ & DM9DDB & $2.461 .407,84$ \\
\hline $\mathbf{1 1}$ & JWT & $2.419 .039,06$ \\
\hline $\mathbf{1 2}$ & DPZET & $2.346 .062,72$ \\
\hline $\mathbf{1 3}$ & MY PROPAGANDA & $2.236 .877,18$ \\
\hline $\mathbf{1 4}$ & FCB BRASIL & $2.041 .788,88$ \\
\hline $\mathbf{1 5}$ & LEW LARA TBWA & $1.799 .283,32$ \\
\hline $\mathbf{1 6}$ & NBS & $1.584 .500,32$ \\
\hline $\mathbf{1 7}$ & LODUCCA & $1.517 .334,94$ \\
\hline $\mathbf{1 8}$ & PROPEG & $1.500 .403,29$ \\
\hline $\mathbf{1 9}$ & ARTPLAN & $1.480 .759,65$ \\
\hline $\mathbf{2 0}$ & TALENT MARCEL & $1.468 .912,77$ \\
\hline $\mathbf{2 1}$ & F NAZCASES & $1.457 .005,06$ \\
\hline $\mathbf{2 2}$ & HEADS PROPAGANDA & $1.379 .180,40$ \\
\hline $\mathbf{2 3}$ & MULTI SOLUTION & $1.264 .085,80$ \\
\hline $\mathbf{2 4}$ & NOVA SB & $1.243 .304,56$ \\
\hline $\mathbf{2 5}$ & ESCALA COMUNICAÇAO & $1.227 .896,28$ \\
\hline $\mathbf{2 6}$ & GREY & $1.215 .559,85$ \\
\hline $\mathbf{2 7}$ & NEOGAMA & $1.195 .085,69$ \\
\hline $\mathbf{2 8}$ & PANDORA PUBLICIDADE & $1.159 .010,11$ \\
\hline $\mathbf{2 9}$ & E MIDIA PROPAGANDA E MERKETING & $1.081 .976,02$ \\
\hline $\mathbf{3 0}$ & AGENCIA WE & $1.069 .501,16$ \\
\hline $\mathbf{3 1}$ & I BRASIL COMUNICAÇAO & $913.097,13$ \\
\hline $\mathbf{3 2}$ & Z MAIS & $896.914,60$ \\
\hline $\mathbf{3 3}$ & MARTER ROMA WAITEMAN & $825.162,15$ \\
\hline $\mathbf{3 4}$ & RINO PUBLICIDADE & $762.478,79$ \\
\hline $\mathbf{3 5}$ & FISCHER & $753.652,73$ \\
\hline & & \\
\hline & & \\
\hline
\end{tabular}




\begin{tabular}{|c|l|c|}
\hline RANKING & \multicolumn{1}{|c|}{ AGENCIA } & INVERSIÓN (R\$) \\
\hline $\mathbf{3 6}$ & FULLPACK COMUNICAÇAO & $733.082,99$ \\
\hline $\mathbf{3 7}$ & WIEDEN KENNEDY & $706.128,33$ \\
\hline $\mathbf{3 8}$ & REF COMUNICAÇAO & $632.759,57$ \\
\hline $\mathbf{3 9}$ & MOMA PORPAGANDA & $570.298,54$ \\
\hline $\mathbf{4 0}$ & IMPACTO PROPAGANDA & $536.127,84$ \\
\hline $\mathbf{4 1}$ & SINERGIA PUBLICIDADE & $521.448,79$ \\
\hline $\mathbf{4 2}$ & 3a WORLDWIDE SOUTH AMERICA & $462.229,48$ \\
\hline $\mathbf{4 3}$ & DENTSU & $420.848,85$ \\
\hline $\mathbf{4 4}$ & EUGENIO PUBLICIDADE & $420.647,81$ \\
\hline $\mathbf{4 5}$ & MCGARRY BOWEN & $394.574,90$ \\
\hline $\mathbf{4 6}$ & REPENSE COMUNICAÇAO & $387.084,26$ \\
\hline $\mathbf{4 7}$ & PANDA AGENCIA & $375.039,09$ \\
\hline $\mathbf{4 8}$ & SANTA CLARA & $348.575,95$ \\
\hline $\mathbf{4 9}$ & WUNDERMAN & $315.102,16$ \\
\hline $\mathbf{5 0}$ & OBJETIVO PROPAGANDA & $310.163,65$ \\
\hline
\end{tabular}

Fuente: Elaboración propia.

La publicidad en Brasil es un tema novedoso, siguiendo la tendencia general de la región iberoamericana (NÚÑEZ; GONZÁLEZ, 2016). Sin embargo, se debe tener en cuenta que, a pesar de ello, Brasil ha sabido posicionarse como uno de los referentes en sector publicitario dentro del área Iberoamericana.

\section{METOdOLOGÍA DE LA INVESTIGACIÓN}

Este trabajo tiene como objetivo principal, analizar la posición de las agencias brasileñas en el ranking Iberoamérica de publicidad, para el periodo comprendido entre 2014 y 2016, comparando el top 50 de agencias para los tres años. El tamaño muestral para dicho periodo es de 113, 114 y 71 agencias publicitarias respectivamente.

El Ranking Iberoamericano de Publicidad evalúa a las agencias según su calidad y a través de un algoritmo que pondera tanto los premios con los que las agencias son galardonadas por sus trabajos publicitarios en festivales. Previamente a la elaboración del ranking, se han dividido los festivales en cuatro categorías, que se pueden subdividir en dos, nacional e internacional. Para la categoría nacional están nacional (que incluye festivales que fueron bajamente puntuados por profesionales del sector) y nacional+ (que incluye 
festivales más importantes). Por otro lado, para la categoría internacional se encuentran internacional (el cual incluye principalmente festivales europeos y latinoamericanos) e internacional+ (que recoge festivales de índole mundial).

Para la ponderación de premios se ha seguido una valoración similar. El valor 1 ha sido dado a aquellos premios de mayor categoría (como Gran Premio, Oro, ...) mientras que al resto se les ha dado valores de 2, 3, 4 y 5 según disminuye la categoría en la que han sido premiados. Para aquellos festivales que entregan premios extraordinarios, como por ejemplo el premio Platino del Festival el Sol (que refleja el mejor anuncio de todas las categorías), se le ha otorgado una ponderación de 0,5.

Tal y como se puede ver en la siguiente fórmula, el cálculo del valor de las agencias es normalizado ya que para crear el ranking de un año se tienen en cuenta los premios recibidos en las dos ediciones anteriores. En la siguiente fórmula, obtenida del trabajo de Repiso, Llorente-Barroso y García-García (2013), se puede ver cómo sería la fórmula para el 2014, que es el primer año analizado en este trabajo.

$$
\operatorname{Agencia~}_{z}=\frac{\sum_{i=1}^{m} \sum_{j=1}^{n} \frac{F_{i}}{A n_{j}}+\sum_{x=1}^{p} \sum_{y=1}^{q} \frac{F_{x}}{A n_{y}}}{\left(\text { Agencia }_{\alpha}\right)^{1 / 2}}
$$

Valor normalizado de la agencia " $z$ " para el periodo 2014:

z: agencia evaluada

m: festival

n: posición del anuncio dentro del festival

$F_{i}$ : valor del festival en 2012

$A n_{j}$ : valoración del premio recibido en el festival en 2012

p: festival

q: posición del anuncio dentro del festival

$F_{x}$ : valor del festival en 2013

$A n_{y}$ : valoración del premio recibido en el festival en 2013 


\section{RESULTADOS DE LA INVESTIGACIÓN}

A continuación, se analizan las posiciones de las agencias de publicidad de Brasil según el Ranking Iberoamericano de Publicidad para los años 2014,2015 y 2016. Una vez ponderados los premios según la metodología anteriormente explicada, las Tablas 5, 6 y 7 muestran el top 50 de las agencias para el periodo seleccionado.

Tabla 4 - Top 50 agencias publicitarias en Brasil para el año 2014

\begin{tabular}{|c|l|c|}
\hline $\begin{array}{c}\text { POSICIÓN } \\
\mathbf{2 0 1 4}\end{array}$ & \multicolumn{1}{|c|}{ NOMBRE } & $\begin{array}{c}\text { PUNTUACIÓN } \\
\mathbf{2 0 1 4}\end{array}$ \\
\hline $\mathbf{1}$ & OGILVY\&MATHER & 1000 \\
\hline $\mathbf{2}$ & ALMAPBBDO BRASIL & 966 \\
\hline $\mathbf{3}$ & F/NAZCA SAATCHI\&SATCHI & 678 \\
\hline $\mathbf{4}$ & LEO BURNETT TAILOR MADE & 498 \\
\hline $\mathbf{5}$ & DDB BRASIL & 488 \\
\hline $\mathbf{6}$ & LODUCCA & 467 \\
\hline $\mathbf{7}$ & YOUNG \& RUBICAM BRASIL & 443 \\
\hline $\mathbf{8}$ & GIOVANNI+DRAFTFCB & 397 \\
\hline $\mathbf{9}$ & JWT BRASIL & 341 \\
\hline $\mathbf{1 0}$ & PUBLICIS BRASIL & 260 \\
\hline $\mathbf{1 1}$ & FIGER 36O & 241 \\
\hline $\mathbf{1 2}$ & ISOBAR & 219 \\
\hline $\mathbf{1 2}$ & DIM\&CANZIAN & 219 \\
\hline $\mathbf{1 4}$ & BFERRAZ & 215 \\
\hline $\mathbf{1 5}$ & TALENT & 184 \\
\hline $\mathbf{1 6}$ & INNOVA & 183 \\
\hline $\mathbf{1 7}$ & BORGHIERH/LOWE & 181 \\
\hline $\mathbf{1 8}$ & FUTURE GROUP & 176 \\
\hline $\mathbf{1 9}$ & FISCHER & 174 \\
\hline $\mathbf{2 0}$ & DM9/DDB & 173 \\
\hline $\mathbf{2 1}$ & LEW'LARATBWA & 167 \\
\hline $\mathbf{2 1}$ & ARTPLAN & 167 \\
\hline $\mathbf{2 3}$ & OPEN AND PARTNERS & 156 \\
\hline $\mathbf{2 4}$ & SANTA CLARA & 151 \\
\hline $\mathbf{2 5}$ & WIEDEN+KENNEDY & 146 \\
\hline $\mathbf{2 6}$ & HOT SHOP COMUNICAÇAO & 144 \\
\hline $\mathbf{2 7}$ & CASE IMAGINE & 141 \\
\hline $\mathbf{2 8}$ & WUNDERMAN BRASIL & 137 \\
\hline $\mathbf{2 9}$ & R/GREENBERG ASSOCIATES PUBLICIDADE LTDA & 136 \\
\hline $\mathbf{3 0}$ & AFRICA & 134 \\
\hline & & \\
\hline & & 2 \\
\hline
\end{tabular}




\begin{tabular}{|c|l|c|}
\hline $\begin{array}{c}\text { POSICIÓN } \\
\mathbf{2 0 1 4}\end{array}$ & \multicolumn{1}{|c|}{ NOMBRE } & $\begin{array}{c}\text { PUNTUACIÓN } \\
\mathbf{2 0 1 4}\end{array}$ \\
\hline $\mathbf{3 1}$ & GREY & 132 \\
\hline $\mathbf{3 2}$ & WMCCANN & 129 \\
\hline $\mathbf{3 2}$ & REVOLUTION & 129 \\
\hline $\mathbf{3 4}$ & EDITORA ABRIL & 122 \\
\hline $\mathbf{3 5}$ & ONE DIGITAL & 116 \\
\hline $\mathbf{3 5}$ & TÁTIL DESIGN & 116 \\
\hline $\mathbf{3 5}$ & GREY141 & 116 \\
\hline $\mathbf{3 8}$ & UMBIGO DO MUNDO & 112 \\
\hline $\mathbf{3 9}$ & GRECO DESIGN & 108 \\
\hline $\mathbf{4 0}$ & NBS & 106 \\
\hline $\mathbf{4 0}$ & ATIVA & 106 \\
\hline $\mathbf{4 0}$ & OGILVYONE BRASIL COMUNICACAO LTDA & 106 \\
\hline $\mathbf{4 3}$ & MIAMI AD SCHOOL/ESPM & 100 \\
\hline $\mathbf{4 4}$ & NETZA PROMOÇOES & 95 \\
\hline $\mathbf{4 5}$ & NEWSTYLE & 91 \\
\hline $\mathbf{4 6}$ & MEDIA CONTACTS & 86 \\
\hline $\mathbf{4 6}$ & NARITA DESIGN & 86 \\
\hline $\mathbf{4 8}$ & PONCE & 82 \\
\hline $\mathbf{4 8}$ & ATENTO & 82 \\
\hline $\mathbf{4 8}$ & THE WALT DISNEY COMPANY LATIN AMERICA & 82 \\
\hline
\end{tabular}

Fuente: Elaboración propia

Tabla 5 - Top 50 agencias publicitarias en Brasil para el año 2015

\begin{tabular}{|c|l|c|}
\hline $\begin{array}{c}\text { POSICIÓN } \\
\mathbf{2 0 1 5}\end{array}$ & \multicolumn{1}{|c|}{ NOMBRE } & $\begin{array}{c}\text { PUNTUACIÓN } \\
\mathbf{2 0 1 5}\end{array}$ \\
\hline $\mathbf{1}$ & ALMAPBBDO BRASIL & 1000 \\
\hline $\mathbf{2}$ & OGILVY\&MATHER & 954 \\
\hline $\mathbf{3}$ & LEO BURNETT TAILOR MADE & 656 \\
\hline $\mathbf{4}$ & F/NAZCA SAATCHI\&SAATCHI & 608 \\
\hline $\mathbf{5}$ & LODUCCA & 523 \\
\hline $\mathbf{6}$ & DDB BRASIL & 478 \\
\hline $\mathbf{7}$ & YOUNG \& RUBICAM BRASIL & 436 \\
\hline $\mathbf{8}$ & FCB BRASIL & 348 \\
\hline $\mathbf{9}$ & GIOVANNI+DRAFTFCB & 342 \\
\hline $\mathbf{1 0}$ & JWT BRASIL & 325 \\
\hline $\mathbf{1 1}$ & BORGHIERH/LOWE & 310 \\
\hline $\mathbf{1 2}$ & ISOBAR & 256 \\
\hline $\mathbf{1 3}$ & DIM\&CANZIAN & 252 \\
\hline $\mathbf{1 4}$ & INNOVA & 245 \\
\hline
\end{tabular}




\begin{tabular}{|c|c|c|}
\hline $\begin{array}{c}\text { POSICIÓN } \\
2015\end{array}$ & NOMBRE & $\begin{array}{c}\text { PUNTUACIÓN } \\
2015\end{array}$ \\
\hline 15 & PUBLICIS BRASIL & 237 \\
\hline 16 & DM9/DDB & 222 \\
\hline 17 & CASE IMAGINE & 204 \\
\hline 18 & FIGER 360 & 198 \\
\hline 18 & PEPPER & 198 \\
\hline 20 & BLUEHIVE BRASIL & 188 \\
\hline 21 & ARTPLAN & 186 \\
\hline 22 & GREY141 & 181 \\
\hline 23 & AFRICA & 177 \\
\hline 24 & WUNDERMAN BRASIL & 163 \\
\hline 25 & R/GREENBERG ASSOCIATES PUBLICIDADE LTDA & 159 \\
\hline 26 & FISCHER & 154 \\
\hline 27 & MARKETDATA & 154 \\
\hline 28 & REVOLUTION & 152 \\
\hline 29 & WIEDEN+KENNEDY & 145 \\
\hline 30 & LEW'LARATBWA & 143 \\
\hline 31 & GREY & 142 \\
\hline 32 & HOT SHOP COMUNICAÇAO & 127 \\
\hline 33 & TALENT & 126 \\
\hline 34 & NETZA PROMOÇOES & 124 \\
\hline 35 & FUTURE GROUP & 121 \\
\hline 36 & SANTA CLARA & 119 \\
\hline 37 & A VERA! & 113 \\
\hline 38 & HAVAS DIGITAL & 102 \\
\hline 38 & OPEN AND PARTNERS & 102 \\
\hline 40 & ATENTO & 97 \\
\hline 41 & GRECO DESIGN & 92 \\
\hline 42 & AGÊNCIA MOOD & 90 \\
\hline 42 & NBS & 90 \\
\hline 44 & GLOBAL & 88 \\
\hline 44 & PBC COMUNICAÇAO LTDA & 88 \\
\hline 44 & THE WALT DISNEY COMPANY LATIN AMERICA & 88 \\
\hline 47 & FOX INTERNATIONAL & 83 \\
\hline 47 & MEDIA CONTACTS & 83 \\
\hline 49 & ALMACÉN & 80 \\
\hline 49 & E/OU MKT DE RELACIONAMENTO & 80 \\
\hline
\end{tabular}

Fuente: Elaboración propia 
Tabla 6- Top 50 agencias publicitarias en Brasil para el año 2016

\begin{tabular}{|c|l|c|}
\hline $\begin{array}{c}\text { POSICIÓN } \\
\mathbf{2 0 1 6}\end{array}$ & \multicolumn{1}{|c|}{ NOMBRE } & $\begin{array}{c}\text { PUNTUACIÓN } \\
\mathbf{2 0 1 6}\end{array}$ \\
\hline $\mathbf{1}$ & ALMAPBBDO BRASIL & 1000 \\
\hline $\mathbf{2}$ & LEO BURNETT TAILOR MADE & 807 \\
\hline $\mathbf{3}$ & OGILVY\&MATHER & 666 \\
\hline $\mathbf{4}$ & FCB BRASIL & 633 \\
\hline $\mathbf{5}$ & LODUCCA & 555 \\
\hline $\mathbf{6}$ & F/NAZCA SAATCHI\&SAATCHI & 537 \\
\hline $\mathbf{7}$ & BORGHIERH/LOWE & 448 \\
\hline $\mathbf{8}$ & YOUNG \& RUBICAM BRASIL & 404 \\
\hline $\mathbf{9}$ & DDB BRASIL & 364 \\
\hline $\mathbf{1 0}$ & PEPPER & 360 \\
\hline $\mathbf{1 1}$ & BLUEHIVE BRASIL & 342 \\
\hline $\mathbf{1 2}$ & JWT BRASIL & 329 \\
\hline $\mathbf{1 3}$ & DM9/DDB & 323 \\
\hline $\mathbf{1 4}$ & ISOBAR & 319 \\
\hline $\mathbf{1 5}$ & DIM\&CANZIAN & 305 \\
\hline $\mathbf{1 6}$ & MARKETDATA & 281 \\
\hline $\mathbf{1 7}$ & WUNDERMAN BRASIL & 268 \\
\hline $\mathbf{1 8}$ & INNOVA & 266 \\
\hline $\mathbf{1 9}$ & CASE IMAGINE & 249 \\
\hline $\mathbf{1 9}$ & GIOVANNI+DRAFTFCB & 249 \\
\hline $\mathbf{2 1}$ & LEW'LARATBWA & 244 \\
\hline $\mathbf{2 2}$ & GREY141 & 241 \\
\hline $\mathbf{2 2}$ & PUBLICIS BRASIL & 241 \\
\hline $\mathbf{2 4}$ & AFRICA & 226 \\
\hline $\mathbf{2 5}$ & FUTURE GROUP & 219 \\
\hline $\mathbf{2 6}$ & A VERA! & 131 \\
\hline $\mathbf{2 6}$ & WIEDEN+KENNEDY & 196 \\
\hline $\mathbf{2 8}$ & TALENT & 184 \\
\hline $\mathbf{2 9}$ & HAVAS DIGITAL & 130 \\
\hline $\mathbf{3 0}$ & GLOBAL & 151 \\
\hline $\mathbf{3 1}$ & FISCHER & 146 \\
\hline $\mathbf{3 1}$ & FOX INTERNATIONAL & 206 \\
\hline $\mathbf{3 3}$ & R/GREENBERG ASSOCIATES PUBLICIDADE LTDA & \\
\hline $\mathbf{3 4}$ & CHEIL BRASIL & 131 \\
\hline $\mathbf{3 5}$ & MARCATIVA COMUNICAÇÃO ESTRATÉGICA LTDA & \\
\hline & & 249 \\
\hline
\end{tabular}




\begin{tabular}{|c|l|c|}
\hline $\begin{array}{c}\text { POSICIÓN } \\
\mathbf{2 0 1 6}\end{array}$ & \multicolumn{1}{|c|}{ NOMBRE } & $\begin{array}{c}\text { PUNTUACIÓN } \\
\mathbf{2 0 1 6}\end{array}$ \\
\hline $\mathbf{3 5}$ & NETZA PROMOÇOES & 131 \\
\hline $\mathbf{3 5}$ & ONEWG & 131 \\
\hline $\mathbf{3 5}$ & OPEN AND PARTNERS & 131 \\
\hline $\mathbf{3 5}$ & PBC COMUNICAÇAO LTDA & 131 \\
\hline $\mathbf{3 5}$ & PROJECT1 & 131 \\
\hline $\mathbf{4 1}$ & ARTPLAN & 128 \\
\hline $\mathbf{4 2}$ & F BIZ & 119 \\
\hline $\mathbf{4 2}$ & GOOGLE BRASIL & 119 \\
\hline $\mathbf{4 2}$ & PAIM COMUNICAÇÃO & 119 \\
\hline $\mathbf{4 5}$ & DDB LATINA & 113 \\
\hline $\mathbf{4 5}$ & REVOLUTION & 113 \\
\hline $\mathbf{4 7}$ & ARANDAS & 107 \\
\hline $\mathbf{4 8}$ & A VOZ DO BRASIL & 93 \\
\hline $\mathbf{4 8}$ & AGÊNCIA MOOD & 93 \\
\hline $\mathbf{4 8}$ & BEGIANT ADVERTAINMENT & 93 \\
\hline $\mathbf{4 8}$ & DM9SUL & 93 \\
\hline
\end{tabular}

Fuente: Elaboración propia

Analizando las agencias publicitarias de Brasil, se observa como ALMAPBBDO Brasil encabeza el ranking durante dos años consecutivos, en 2015 y 2016 con una puntuación de 1000, el máximo que se puede obtener en este ranking. Solo cede su primera posición en 2014, año en el cual el primer puesto es ocupado por Ogilvy\&Mather, que se sitúa en segunda y tercera posición en la lista para los dos años siguientes respectivamente. Leo Burnett Tailor Made es otra de las agencias que ocupa las primeras posiciones durante los tres años, situándose en el cuarto puesto en el año 2014, tercera posición para el 2015 y segunda para el 2016.

Loducca consigue mantenerse casi contante en el quinto puesto durante todo el periodo, exceptuando el primer año analizado, 2014, para el cual obtuvo la sexta posición. Cercana a la posición de estas agencias se encuentra F/Nazca Saatchi\&Saatchi la cual se situó en tercera, cuarta y sexta posición respectivamente para cada uno de los años, perdiendo por lo tanto tres posiciones con respecto al primer año analizado. 
Esta última agencia mencionada no es la única que pierde puestos con respecto al comienzo del periodo analizado. DDB Brasil pasa del puesto 5 al 9. Young \& Rubicam Brasil que comienza en el séptimo puesto, baja uno para el último año. Giovanni+DRAFTFCB pasa del 8 al 19. JWT Brasil del 9 al 12. Publicis Brasil tiene una notable caída, empezando en el puesto 10 y terminando en el 22. Future Group va del 18 al 25. Isobar y Dim\&Canzian, ambas en el puesto 12, pasan al 14 y 15 respectivamente.

En la posición contraria se encuentran aquellas agencias que han subido puestos, como Borghierh/Lowe que empieza en puesto 17 y acaba en el 7. DM9/DDB pasa del 20 al 13. Case Imagine se alza del puesto 27 al 19. Grey141 sube del 35 al 22. Africa también aumenta su posición del 30 al 24.

Son una minoría las agencias que se mantienen en el mismo puesto que en el que empezaron, siendo una de ellas Lew'Laratbwa, en la posición 21.

\section{CONCLUSIONES}

La tendencia actual en Iberoamérica por parte de las agencias publicitarias es que se tiende a la internacionalización. Este tipo de agencias representa una nueva forma de comunicación que es tan importante para las compañías como para sus otros activos (FEJES, 1980). Tal y como señalan Merino y Repiso (2015), la estrategia de estas compañías internacionales se centra en adaptar el conocimiento y el contenido desde la empresa matriz hacia las sedes situadas en el extranjero. Hay tres habilidades reflejadas en este proceso: eficiencia y competitividad a escala global, reacción y flexibilidad en el ámbito nacional y la capacidad de cruzar distintos mercados para utilizar el aprendizaje de manera global (BARTLETT; GHOSHAL, 2003).

Con respecto a las compañías de publicidad, Merino y Repiso (2015) presentan una teoría sobre las agencias de publicidad colombianas. Su investigación se guía por la hipótesis de que las compañías transnacionales deben servir como herramienta de introducción de productos extranjeros al mercado autóctono, mientras que las agencias nacionales deben servir a las empresas locales.

Durante este trabajo se ha intentado dar una visión más clara sobre la publicidad en Brasil. Para ello se ha llevado a cabo un análisis de las 50 agencias más creativas de Brasil. 
ALMAPBBDO Brasil se posiciona como la mejor agencia, encabezando el ranking durante dos años consecutivos, en 2015 y 2016. Solo cede su primera posición en 2014, año en el cual el primer puesto es ocupado por Ogilvy\&Mather, que se sitúa como la segunda mejor agencia con la segunda y tercera posición en la lista para los dos años siguientes respectivamente. Leo Burnett Tailor Made es otra de las agencias que ocupa las primeras posiciones durante los tres años, colocándose entre las cinco mejores del periodo junto con Loducca y F/Nazca Saatchi\&Saatchi.

Si se comparan estos resultados con la tabla 5, que muestra la inversión de las empresas de publicidad, se observa que ésta no es una característica decisiva que indique que las empresas que más invierten son también las más creativas. Solo cuatro empresas de las diez que más dinero invierten son también las más creativas de Brasil: ALMAPBBDO Brasil, Leo Burnett Tailor Made, Ogilvy\&Mather y Young \&Rubicam.

\section{REFERENCIAS}

ASSOCIAÇÃO BRASILEIRA DE AGÊNCIAS DE PUBLICIDADE (ABAP). 2016. Disponible en: <http://www.abap.com.br/index.cfm>. Acceso en: 13 abr. 2017.

BARTLETT, Christopher; GHOSHAL, Sumantra. ¿Qué es un gerente global? Harvard Business Review, Allston, v. 81, n. 8, p. 87-94, 2003.

FEJES, Fred. The growth of multinational advertising agencies in Latin America. Journal of Communication, Hoboken, v. 30, n. 4, p. 36-49, 1980.

FOREIT, Karen. G.; DE CASTRO, Marcos Paulo; FRANCO, Eliane. The impact of mass media advertising on a voluntary sterilization program in Brazil. Studies in family planning, Nueva York, v. 20, n. 2, p. 107-16, mar./abr. 1989.

MERINO, Adoración; REPISO, Rafael. Las agencias colombianas de publicidad como agentes del marketing nacional e internacional. Anagramas - Rumbos y sentidos de la comunicación, Medellín, v. 14, n. 28, p. 51-66, 2015.

NúÑEZ, Estela; GONZÁLEZ, Almudena. Ranking de publicidad en Iberoamérica ¿cuáles son las principales agencias? Opción, Zulia, v. 32, número especial 8, p. 361-72, 2016.

REPISO, Rafael; BERLANGA, Inmaculada. Ranking UNIR, un proyecto de clasificación de la mejor publicidad iberoamericana. El Profesional de la Información, Barcelona, Espanha, v. 24, n. 4, p. 479-84, 2015. 
REPISO, Rafael; LLORENTE-BARROSO, Carmen, GARCÍA-GARCÍA, Francisco. Ranking ESCO de agencias de publicidad: descripción y resultados. El Profesional de la Información, Barcelona, Espanha, v. 22, n. 2, p. 181-7, mar./abr. 2013.

STATISTA. Advertising industry in Latin America. 2017. Disponible en: <https://www. statista.com/topics/1499/advertising-in-latin-america/>. Acceso en: 10 jul. 2017. 